\title{
Products in Categories without Uniqueness of cod and dom ${ }^{1}$
}

\author{
Artur Korniłowicz \\ Institute of Informatics \\ University of Białystok \\ Sosnowa 64, 15-887 Białystok \\ Poland
}

\begin{abstract}
Summary. The paper introduces Cartesian products in categories without uniqueness of cod and dom. It is proven that set-theoretical product is the product in the category Ens [7].
\end{abstract}

MML identifier: ALTCAT_5, version: $\underline{8.0 .015 .3 .1162}$

The papers [10], [6], [1], [8], [2], [3], [4], [9], [12], [11], and [5] provide the terminology and notation for this paper.

In this paper $I$ denotes a set and $E$ denotes a non empty set.

Let us mention that every binary relation which is empty is also $\emptyset$-defined.

Let $C$ be a graph. We say that $C$ is functional if and only if:

(Def. 1) For all objects $a, b$ of $C$ holds $\langle a, b\rangle$ is functional.

Let us consider $E$. One can verify that $\operatorname{Ens}_{E}$ is functional.

Let us observe that there exists a category which is functional and strict.

Let $C$ be a functional category structure. One can verify that the graph of $C$ is functional.

Let us observe that there exists a graph which is functional and strict.

Let us note that there exists a category which is functional and strict.

Let $C$ be a functional graph and let $a, b$ be objects of $C$. Observe that $\langle a, b\rangle$ is functional.

\footnotetext{
${ }^{1}$ This work has been supported by the Polish Ministry of Science and Higher Education project "Managing a Large Repository of Computer-verified Mathematical Knowledge" (N N519 385136).

(C) 2012 University of Białystok CC-BY-SA License ver. 3.0 or later ISSN $1426-2630(\mathrm{p}), 1898-9934(\mathrm{e})$
} 
Let $C$ be a non empty category structure and let $I$ be a set. An objects family of $I$ and $C$ is a function from $I$ into $C$.

Let $C$ be a non empty category structure, let $o$ be an object of $C$, let $I$ be a set, and let $f$ be an objects family of $I$ and $C$. A many sorted set indexed by $I$ is said to be a morphisms family of $o$ and $f$ if:

(Def. 2) For every set $i$ such that $i \in I$ there exists an object $o_{1}$ of $C$ such that $o_{1}=f(i)$ and $\operatorname{it}(i)$ is a morphism from $o$ to $o_{1}$.

Let $C$ be a non empty category structure, let $o$ be an object of $C$, let $I$ be a non empty set, and let $f$ be an objects family of $I$ and $C$. Let us note that the morphisms family of $o$ and $f$ can be characterized by the following (equivalent) condition:

(Def. 3) For every element $i$ of $I$ holds it $(i)$ is a morphism from $o$ to $f(i)$.

Let $C$ be a non empty category structure, let $o$ be an object of $C$, let $I$ be a non empty set, let $f$ be an objects family of $I$ and $C$, let $M$ be a morphisms family of $o$ and $f$, and let $i$ be an element of $I$. Then $M(i)$ is a morphism from $o$ to $f(i)$.

Let $C$ be a functional non empty category structure, let $o$ be an object of $C$, let $I$ be a set, and let $f$ be an objects family of $I$ and $C$. Observe that every morphisms family of $o$ and $f$ is function yielding.

Next we state the proposition

(1) Let $C$ be a non empty category structure, $o$ be an object of $C$, and $f$ be an objects family of $\emptyset$ and $C$. Then $\emptyset$ is a morphisms family of $o$ and $f$.

Let $C$ be a non empty category structure, let $I$ be a set, let $A$ be an objects family of $I$ and $C$, let $B$ be an object of $C$, and let $P$ be a morphisms family of $B$ and $A$. We say that $P$ is feasible if and only if:

(Def. 4) For every set $i$ such that $i \in I$ there exists an object $o$ of $C$ such that $o=A(i)$ and $P(i) \in\langle B, o\rangle$.

Let $C$ be a non empty category structure, let $I$ be a non empty set, let $A$ be an objects family of $I$ and $C$, let $B$ be an object of $C$, and let $P$ be a morphisms family of $B$ and $A$. Let us observe that $P$ is feasible if and only if:

(Def. 5) For every element $i$ of $I$ holds $P(i) \in\langle B, A(i)\rangle$.

Let $C$ be a category, let $I$ be a set, let $A$ be an objects family of $I$ and $C$, let $B$ be an object of $C$, and let $P$ be a morphisms family of $B$ and $A$. We say that $P$ is projection morphisms family if and only if the condition (Def. 6) is satisfied.

(Def. 6) Let $X$ be an object of $C$ and $F$ be a morphisms family of $X$ and $A$. Suppose $F$ is feasible. Then there exists a morphism $f$ from $X$ to $B$ such that

(i) $f \in\langle X, B\rangle$, 
(ii) for every set $i$ such that $i \in I$ there exists an object $s_{1}$ of $C$ and there exists a morphism $P_{1}$ from $B$ to $s_{1}$ such that $s_{1}=A(i)$ and $P_{1}=P(i)$ and $F(i)=P_{1} \cdot f$, and

(iii) for every morphism $f_{1}$ from $X$ to $B$ such that for every set $i$ such that $i \in I$ there exists an object $s_{1}$ of $C$ and there exists a morphism $P_{1}$ from $B$ to $s_{1}$ such that $s_{1}=A(i)$ and $P_{1}=P(i)$ and $F(i)=P_{1} \cdot f_{1}$ holds $f=f_{1}$.

Let $C$ be a category, let $I$ be a non empty set, let $A$ be an objects family of $I$ and $C$, let $B$ be an object of $C$, and let $P$ be a morphisms family of $B$ and $A$. Let us observe that $P$ is projection morphisms family if and only if the condition (Def. 7) is satisfied.

(Def. 7) Let $X$ be an object of $C$ and $F$ be a morphisms family of $X$ and $A$. Suppose $F$ is feasible. Then there exists a morphism $f$ from $X$ to $B$ such that

(i) $f \in\langle X, B\rangle$,

(ii) for every element $i$ of $I$ holds $F(i)=P(i) \cdot f$, and

(iii) for every morphism $f_{1}$ from $X$ to $B$ such that for every element $i$ of $I$ holds $F(i)=P(i) \cdot f_{1}$ holds $f=f_{1}$.

Let $C$ be a category, let $A$ be an objects family of $\emptyset$ and $C$, and let $B$ be an object of $C$. Note that every morphisms family of $B$ and $A$ is feasible.

One can prove the following propositions:

(2) Let $C$ be a category, $A$ be an objects family of $\emptyset$ and $C$, and $B$ be an object of $C$. If $B$ is terminal, then there exists a morphisms family of $B$ and $A$ which is empty and projection morphisms family.

(3) For every objects family $A$ of $I$ and Ens 1 and for every object $o$ of Ens $s_{1}$ holds $I \longmapsto \emptyset$ is a morphisms family of $o$ and $A$.

(4) Let $A$ be an objects family of $I$ and $E_{n} s_{1}, o$ be an object of Ens 1 , and $P$ be a morphisms family of $o$ and $A$. If $P=I \longmapsto \emptyset$, then $P$ is feasible and projection morphisms family.

Let $C$ be a category. We say that $C$ has products if and only if the condition (Def. 8) is satisfied.

(Def. 8) Let $I$ be a set and $A$ be an objects family of $I$ and $C$. Then there exists an object $B$ of $C$ such that there exists a morphisms family of $B$ and $A$ which is feasible and projection morphisms family.

Let us note that Ens 1 has products.

One can check that there exists a category which has products.

Let $C$ be a category, let $I$ be a set, let $A$ be an objects family of $I$ and $C$, and let $B$ be an object of $C$. We say that $B$ is $A$-cat product-like if and only if:

(Def. 9) There exists a morphisms family of $B$ and $A$ which is feasible and projection morphisms family. 
Let $C$ be a category with products, let $I$ be a set, and let $A$ be an objects family of $I$ and $C$. One can check that there exists an object of $C$ which is $A$-cat product-like.

Let $C$ be a category and let $A$ be an objects family of $\emptyset$ and $C$. Note that every object of $C$ which is $A$-cat product-like is also terminal.

We now state two propositions:

(5) Let $C$ be a category, $A$ be an objects family of $\emptyset$ and $C$, and $B$ be an object of $C$. If $B$ is terminal, then $B$ is $A$-cat product-like.

(6) Let $C$ be a category, $A$ be an objects family of $I$ and $C$, and $C_{1}, C_{2}$ be objects of $C$. Suppose $C_{1}$ is $A$-cat product-like and $C_{2}$ is $A$-cat productlike. Then $C_{1}, C_{2}$ are iso.

In the sequel $A$ is an objects family of $I$ and $\operatorname{Ens}_{E}$.

Let us consider $I, E, A$. Let us assume that $\prod A \in E$. The functor EnsCatProductObj $A$ yielding an object of Ens $_{E}$ is defined by:

(Def. 10) EnsCatProductObj $A=\prod A$.

Let us consider $I, E, A$. Let us assume that $\prod A \in E$. The functor EnsCatProduct $A$ yields a morphisms family of EnsCatProductObj $A$ and $A$ and is defined by:

(Def. 11) For every set $i$ such that $i \in I$ holds $($ EnsCatProduct $A)(i)=\operatorname{proj}(A, i)$.

We now state four propositions:

(7) If $\prod A \in E$ and $\prod A=\emptyset$, then EnsCatProduct $A=I \longmapsto \emptyset$.

(8) If $\prod A \in E$, then EnsCatProduct $A$ is feasible and projection morphisms family.

(9) If $\prod A \in E$, then EnsCatProductObj $A$ is $A$-cat product-like.

(10) If for all $I, A$ holds $\prod A \in E$, then Ens $_{E}$ has products.

\section{REFERENCES}

[1] Grzegorz Bancerek. König's theorem. Formalized Mathematics, 1(3):589-593, 1990.

[2] Czesław Byliński. Functions and their basic properties. Formalized Mathematics, 1(1):5565, 1990.

[3] Czesław Byliński. Functions from a set to a set. Formalized Mathematics, 1(1):153-164, 1990.

[4] Czesław Byliński. Partial functions. Formalized Mathematics, 1(2):357-367, 1990.

[5] Czesław Byliński. Some basic properties of sets. Formalized Mathematics, 1(1):47-53, 1990.

[6] Beata Madras. Basic properties of objects and morphisms. Formalized Mathematics, 6(3):329-334, 1997.

[7] Zbigniew Semadeni and Antoni Wiweger. Wstęp do teorii kategorii i funktorów, volume 45 of Biblioteka Matematyczna. PWN, Warszawa, 1978.

[8] Andrzej Trybulec. Binary operations applied to functions. Formalized Mathematics, 1(2):329-334, 1990.

[9] Andrzej Trybulec. Many sorted sets. Formalized Mathematics, 4(1):15-22, 1993.

[10] Andrzej Trybulec. Categories without uniqueness of cod and dom. Formalized Mathematics, 5(2):259-267, 1996.

[11] Zinaida Trybulec. Properties of subsets. Formalized Mathematics, 1(1):67-71, 1990. 
[12] Edmund Woronowicz. Relations and their basic properties. Formalized Mathematics, 1(1):73-83, 1990.

Received August 19, 2012 